\title{
POSTER
}

\section{Work and Leisure}

$D-R$. CHEN. Using somatosensory games to improve the health of the elderly in the community. Gerontechnology 2018;17(Suppl):196s; https://doi.org/10.4017/gt.2018.17.s.191.00 Purpose Somatosensory games have become popular in recent years. We attempt to use somatosensory games as health promotion tools for the elderly. The study aims to examine whether somatosensory games can help elder adults in the community enhance physical health and become more socially engaged. Methods The study was a 6-month program using Xbox Kinect in the Wanhua district health center, Taipei, Taiwan. Participants in the experimental group played pre-selected commercially available somatosensory games every Tuesday and Friday. Participants in the control group $(n=26)$ completed a pre/post-test questionnaire and physical fitness tests. After playing somatosensory games, participants of the experimental group $(n=29)$ would take physical fitness tests every month including: (1) Body composition analysis (BCA); (2) Grip strength (hand muscle); (3) 30 second sitting to standing exercise; and (4) 2 min leg lifting. Results \& Discussion No significant demographic differences were found between experimental and control groups. Paired tests indicated that elderly people in the experimental group presented significantly better muscular endurance including grip strength; 30 seconds sitting to standing exercise, and 2 min leg lifting steps. Body flexibility (e.g., arms across stretching behind the back) also significantly increased. Social network structure was measured before and after the intervention. Social cohesion in the experimental group was greatly increased after 6 months. In conclusion, somatosensory games can increase body flexibility and muscle endurance. Yet, social connections were also greatly improved after playing games.

Keywords: somatosensory games, elderly, health promotion Address: National Taiwan University, Taiwan;

E:duan@ntu.edu.tw 